\title{
CLOUD-CAPPED TOWERS: CAPTURING TERRAIN CHARACTERISTICS USING TOPOGRAPHIC FUNCTIONALS
}

\author{
EDWARD EARL $^{1}$, DAVID METZLER ${ }^{2}$ \\ ${ }^{1}$ Independent researcher \\ ${ }^{2}$ Albuquerque Academy, Albuquerque, New Mexico, USA
}

Manuscript received: June 26, 2014

Revised version: September 9, 2015

\begin{abstract}
EarL E., MetZler D., 2015. Cloud-capped towers: Capturing terrain characteristics using topographic functionals. Quaestiones Geographicae 34(4), Bogucki Wydawnictwo Naukowe, Poznań, pp. 7-23, 5 tables, 22 figs. DOI 10.1515/ quageo-2015-0033, ISSN 0137-477X.

AвSTRACT: We introduce three related mathematical measures of terrain based on a particularly elegant combination of relief and steepness, which we term omnidirectional relief and steepness (ORS). One measure, reduced ORS (RORS), is particularly suited to creating lists of notable features, and another, domain relief and steepness (DRS), measures the ruggedness of a region.
\end{abstract}

KEY wORDS: topography, orography, hypsometry, landforms, mathematical measures of terrain

David Metzler, Albuquerque Academy, 6400 Wyoming Blvd NE, Albuquerque, NM 87109, USA; e-mail: metzler@aa.edu

\section{Introduction}

Mountain terrain is more impressive than low, rolling hills or a flat plain. Much of that impressiveness is personal and subjective, but there are commonalities as well. In particular, large relief and steep drops correlate strongly with most people's idea of visual impressiveness. In this paper, we introduce three measures ${ }^{1}$ of mountain terrain based on the idea of relief and steepness. Constructing such a measure turns out to be a bit tricky. Mountains are often measured by their absolute elevation (i.e. above sea level), but that is not directly material to the visual impressiveness of a mountain. A gentle 1000 meter hill high

1 We use the word "measure" throughout in the sense of a measurement of terrain, not in the mathematician's sense of the term. on the Tibetan Plateau could have a summit elevation of well over 6000 meters, higher than anything in North America, but not appear at all impressive. Mathematically, the opposite extreme to looking simply at elevation (i.e. the values of the height function of a landscape) is to look at the derivative of the height function. However, that is not appropriate either, as it focuses only on steepness and not on total relief. In fact, since real terrain has a roughly fractal structure, taking a derivative will measure the variation in slope on the smallest scales. For example, an ordinary curb at the edge of a sidewalk would yield an infinite value for the derivative of the height function, reflecting its vertical nature, but ignoring its tiny overall relief.

Hence we seek measures that simultaneously capture relief and steepness. The first measure we introduce assigns a number to any point on a landscape that captures how highly and steep- 
ly that point rises above surrounding terrain. By using the integral of a certain function, it automatically incorporates both relief and steepness information, averaged over all directions around the given point. We call this measure (or more pretentiously, this topographic functional) omnidirectional relief and steepness (ORS); it was first introduced in Earl and Metzler (2013), and is the foundation for the other two measures introduced in this paper. The first of those is reduced ORS (RORS), which is a modification of ORS that addresses the desire to create a ranked list of relatively "independent" impressive peaks, disregarding minor subpeaks. The second is domain relief and steepness (DRS), which does not assign a number to a specific feature, but to a whole region; it is essentially an average of ORS for all points in the region, and is a measure of the ruggedness of that region, in a way that automatically takes into account the overall relief involved and the steepness of the slopes in the region. A typical application is to compare the ruggedness of different mountain ranges.

In the body of the article we give the basic definitions of each measure, and various illustrative examples, applied to real topography. In the appendix we give a more precise definition of RORS and prove certain important mathematical properties of DRS, continuing the work begun in the first article (Earl, Metzler 2013) on these measures.

\section{ORS}

Let $h(x, y)$ be a bounded function of two variables, thought of as the height function of a landscape. (We do not require $h$ to be continuous, to permit the presence of vertical cliffs ${ }^{2}$.) Consider a point $P$ on this landscape (usually, the summit or another high point on a peak), described by its horizontal (map) location $\mathbf{p}=\left(x_{0}, y_{0}\right)$ and height $h(\mathbf{p})$. We want to assign a number, which we will call the omnidirectional relief and steepness (ORS),

\footnotetext{
We could in principle take into account the curvature of the Earth, but we will see that all of the calculations localize strongly, making it a very good approximation to think of the terrain as rising from a flat plane. Also, we do not treat overhanging cliffs in any special way, since that would vastly complicate the mathematical model.
}

that measures how highly and steeply this point rises above the surrounding terrain. It should give a larger number to a larger peak if the two peaks are comparable in steepness, and it should give a larger number to a steeper peak if the two are comparable in height.

So for example, consider a perfectly conical peak of height $H$, rising from a flat plain with angle of elevation $\theta$. The simplest measure we could define for such a peak would simply be the product of the height and the angle ${ }^{3}$. For simplicity, we actually divide the angle by $90^{\circ}$ :

$$
\text { ORS }(\text { cone })=H \cdot \theta / 90^{\circ}
$$

In particular, a "flagpole" (the limit of the cone shape as $\theta$ approaches $90^{\circ}$ ) of height $H$ gets an ORS value of exactly $H$, and that is the highest possible value of ORS for a feature of total height $H$. A more topographically realistic cone of angle $45^{\circ}$ gets an ORS value of $H / 2$, which is a good benchmark to remember in looking at specific examples.

For a general, not perfectly conical feature, we can still define ORS by capturing a kind of sum of how highly and steeply $P$ rises above every other point in its surroundings. Like any continuous sum, it is calculated using an integral over all those surrounding points. It turns out to be best-behaved if we actually calculate it as the square root of the integral ${ }^{4}$ of a certain quantity. The formula that we give below involves a complicated-looking function, but that function is present in order to guarantee the simple result given above for the perfect cone; see Earl and Metzler (2013).

Definition 1. Let $h(x, y)$ be the height function for a landscape, let $\mathbf{p}=\left(x_{0} y_{0}\right)$ be a point in the plane, and let $h_{0}=h\left(x_{0}, y_{0}\right)$. Let $r$ be the radial coordinate based at $P$, i.e. $r(x, y)$ measures the

Note that we should not use the slope of the side of the peak since that would be unbounded as the sides approached vertical. While such perfect "flagpole" features do not exist in nature, any vertical cliff has at least one such side, and using the slope would cause ORS to become infinite in such cases. The angle is the simplest bounded quantity that measures the steepness of the sides of the cone.

4 This is analogous to taking the root-mean-square (RMS) average of a quantity, as is often done in signal processing and other situations. Here the integral is not truly an average, but the idea is the same. 
(horizontal) distance from $(x, y)$ to $\left(x_{0}, y_{0}\right)$, and let $u(x, y)=\left(h_{0}-h(x, y)\right) / r$. The omnidirectional relief and steepness (ORS) of the point $P$ relative to the landscape $h$ is ${ }^{5}$

$$
\operatorname{ORS}(\mathbf{p} ; h)=\left[\int_{\mathrm{R}^{2}}(f(u(x, y)))^{2} d y d x\right]^{1 / 2}
$$

where $f$ is the function given by ${ }^{6} f(u)=0$ for $u$ $<0$, and for $u>0$,

$$
f(u)=\left[\frac{1}{\Pi^{3}}\left(2 u \arctan u-\ln \left(u^{2}+1\right)-\arctan ^{2} u\right)\right]^{1 / 2}
$$

The function $f$ is monotonically increasing, with graph shown in Fig. 1. It does not do too much violence to the spirit of ORS to think of $f(u)$ as very roughly behaving like $u$ itself $^{7}$.

In Fig. 2, we indicate the picture to have in mind when thinking about ORS. One can think of drawing a line from $\left(\mathbf{p}, h_{0}\right)$ to every point in the surrounding landscape, calculating an "impressiveness" rating $f(u)$ based on the slope $u$ of the line, and then doing an RMS-type sum of those ratings. A larger peak (such as Mount Rainier, shown in the picture) will get substantial values for the slope $u$, and hence $f(u)$, over a large area, giving a high ORS value. A steep but small peak

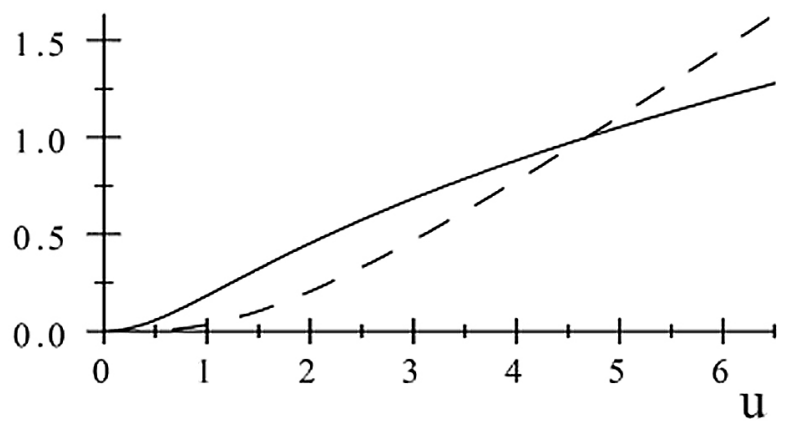

Fig. 1. Plot of $f$ (solid) and $f^{2}$ (dashed)

\footnotetext{
We use single integral signs throughout this paper, in contrast to our use of double integrals for subsets of $\mathrm{R}^{2}$ in the previous paper. This is to avoid cumbersome quadruple integral notation for DRS.

6 You may wonder why $f$ is defined with a square root, which we immediately square away in the integral. That is to present ORS explicitly as an RMS average, which is useful for a theoretical analysis of its properties. The function $f$ also appears in the definition of RORS below, in a way where it is not immediately squared.

7 However, it is crucial that $f(u)$ is of the order of $u^{2}$ and not $u$ for small slopes, or else the integral will not converge.
}

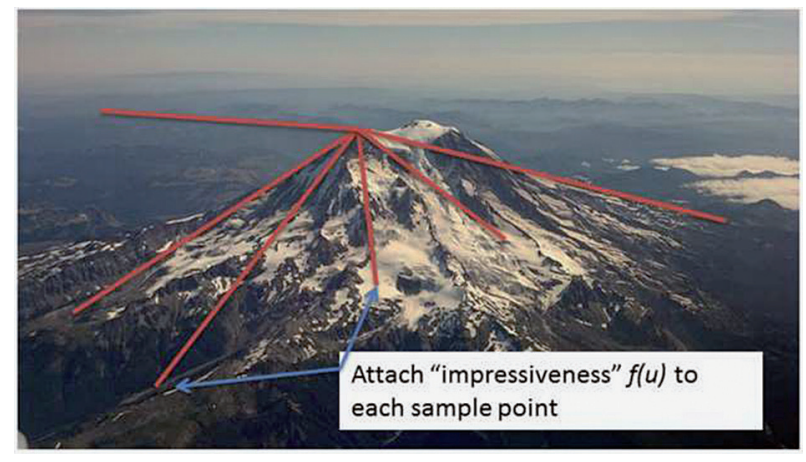

Fig. 2. ORS illustrated by Mount Rainier Source: Wikimedia Commons.

will not get high slope values over such a large area, but it will get very high slopes near the peak, and hence will also get a high ORS value.

In Earl and Metzler (2013) we gave a mathematical analysis of the properties of ORS. Here we will just mention some real-world calculations. For example, the summit of Mount Elbert (Fig. 4), the highest point in the Rocky Mountains, is 4401 meters above sea level [1], while Devils Thumb ${ }^{8}$ (Fig. 3), a striking rock spire on the border between Alaska and British Columbia, rises only to 2767 meters [3]. Based on pure elevation, Elbert far surpasses Devils Thumb. However, Mount Elbert rises from a high base in central Colorado, so its local relief is not nearly as great as its elevation would indicate; nor is it a particularly steep peak. For example, Elbert rises about 1600 meters (one mile) over a horizontal distance of 6.5 kilometers on its southeast flank ${ }^{9}$ - which is not unimpressive. However, the northwest face of Devils Thumb soars an amazing 2000 meters in only $1.6 \mathrm{~km}$, and it is similarly steep in other directions. To get 2000 meters of vertical relief from the summit of Mount Elbert, one has to go about $30 \mathrm{~km}$ away, to the town of Aspen; if one goes $30 \mathrm{~km}$ from Devils Thumb, one gets to tidewater, yielding 2767 meters of relief. It is instructive to peruse the topographical maps for Mount Elbert [2] and for Devils Thumb [4] $]^{10}$. Mount Elbert has

8 There is no apostrophe in the official spelling of the name of this peak.

9 One can verify these numbers using the public-domain mapping website map - per.acme.com, among others.

10 Google Earth produces a good virtual tour of Mount Elbert. However, it has very inaccurate (and misleadingly smoothed-out) elevation data for Devils Thumb. Getting accurate elevation data for steep features in obscure locations, such as Devils Thumb, is one of the challenges of this research. 


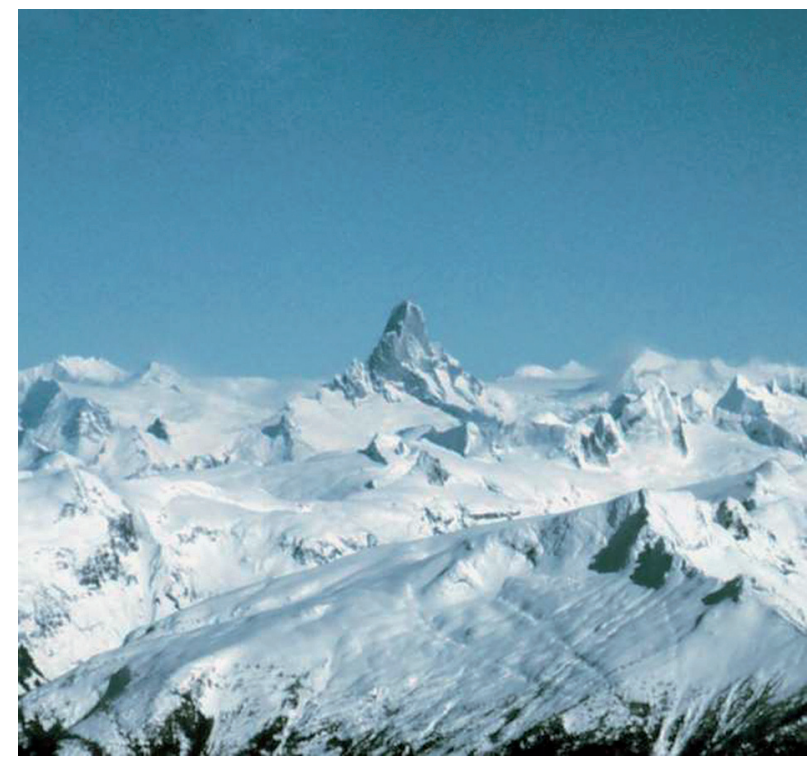

Fig. 3. Devils Thumb, Alaska Source: Wikipedia.

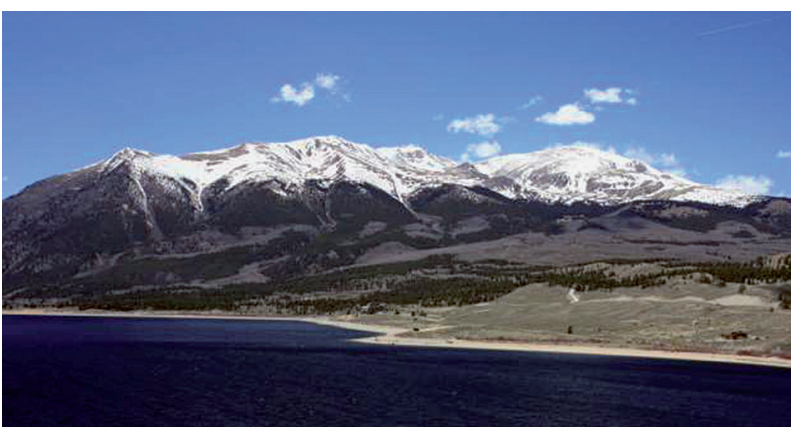

Fig. 4. Mount Elbert, Colorado Source: Wikipedia.

an ORS of 237 meters, while Devils Thumbs' is 828 meters, corresponding to their dramatically different profiles. These values show that a comparison between these two peaks based on ORS gives the opposite result from the comparison suggested by their absolute elevations.

Table 1 lists the six US states with the highest maximum ORS value. Not surprisingly, Alaska tops the list, although Mount McKinley (GE) $(\mathrm{ORS}=1243 \mathrm{~m}$, elev $=6194 \mathrm{~m})$ is not the best point in Alaska. The lower Mount Saint Elias (GE) is very close to tidewater (about $10 \mathrm{~km}$ away, see Fig. 5), and is comparably steep, so it gets a higher ORS value. Most of the other peaks are wellknown, except perhaps Mount Cleveland (GE), the high point of Glacier National Park. (The glaciers there are fast disappearing, but they have carved a number of exceptionally steep peaks.) It is interesting to also compare Mount Whitney (GE), the high point of the contiguous US (ORS = $418 \mathrm{~m}$, elev $=4421 \mathrm{~m}$ ); note that it is bested with-
Table 1. State best points by ORS

\begin{tabular}{|l|r|c|c|}
\hline \multicolumn{1}{|c|}{ Peak } & ORS & Elev & State \\
\hline Mount Saint Elias (GE) & 1334 & 5489 & Alaska \\
\hline Mount Rainier (GE) & 827 & 4392 & Washington \\
\hline Grand Teton (GE) & 683 & 4197 & Wyoming \\
\hline Mount Shasta (GE) & 675 & 4317 & California \\
\hline Mount Cleveland (GE) & 672 & 3190 & Montana \\
\hline Mount Hood (GE) & 649 & 3452 & Oregon \\
\hline
\end{tabular}

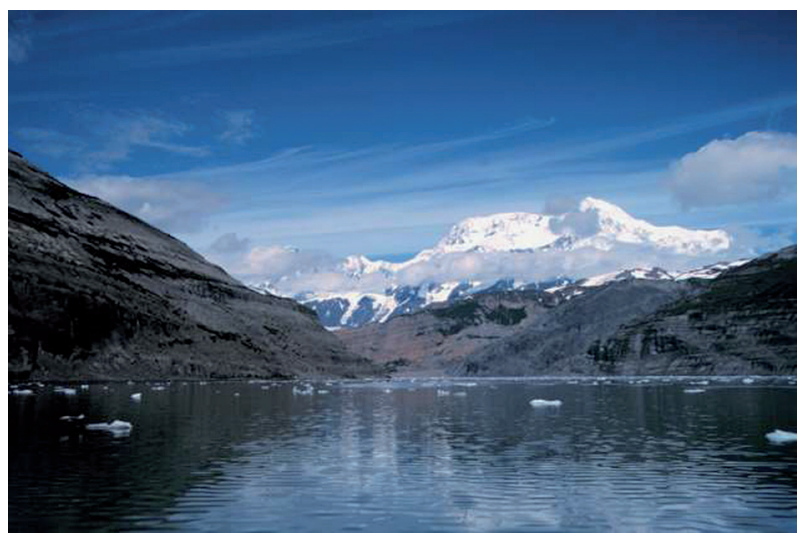

Fig. 5. Mount Saint Elias from Icy Bay Source: Wikimedia Commons.

in California not only by the huge stratovolcano Mount Shasta (GE), but also by Yosemite's El Capitan (575 m) and Half Dome (580 m), among others. As in the case of Mount Elbert, simply being the best in terms of absolute elevation does not guarantee a high rating in terms of ORS.

Worldwide, we have Table $2^{11}$, which lists the top five independent ${ }^{12}$ peaks in the world. Four, including the overall winner Nanga Parbat (Fig. 6), are in the Himalayas, while Rakaposhi is in the nearby Karakoram range. While three of these peaks are in the famed group of fourteen "eight-thousanders" (with elevation over 8000 meters), two are not; in fact Machhapuchhare (Fig. 7) is not even in the top 300 peaks in the world by elevation. (It is a tremendously steep peak, near low terrain, in the Annapurna region of Nepal; it is highly sacred and is off-limits to climbing.) For comparison, Mount Everest, elevation $8848 \mathrm{~m}$, gets a very respectable ORS value of $1302 \mathrm{~m}$. Also note the dramatic difference in scale

11 Since uniform topographic mapping is not available for these peaks, the links are to Google Earth tours. They give the general impression, but be aware that they are not always highly accurate.

12 This list was actually generated by taking the five highest points as ranked by reduced ORS, as in Section 3 , to ensure five truly independent peaks. 
Table 2. World's top five independent peaks by ORS

\begin{tabular}{|l|c|c|c|}
\hline \multicolumn{1}{|c|}{ Peak } & ORS & Elev & State \\
\hline Nanga Parbat & 1740 & 8125 & Pakistan \\
\hline Dhaulagiri & 1680 & 8167 & Nepal \\
\hline Rakaposhi & 1628 & 7788 & Pakistan \\
\hline Machhapuchhare & 1596 & 6993 & Nepal \\
\hline Manaslu & 1550 & 8163 & Nepal \\
\hline
\end{tabular}

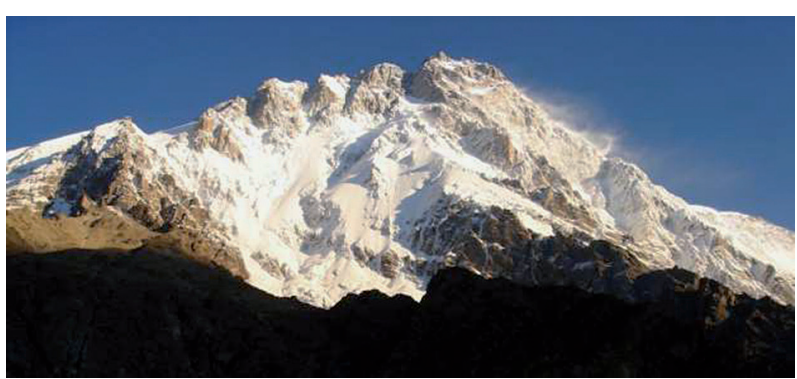

Fig. 6. The southern "Rupal" face of Nanga Parbat, one of the largest mountain faces in the world Source: Wikimedia Commons.

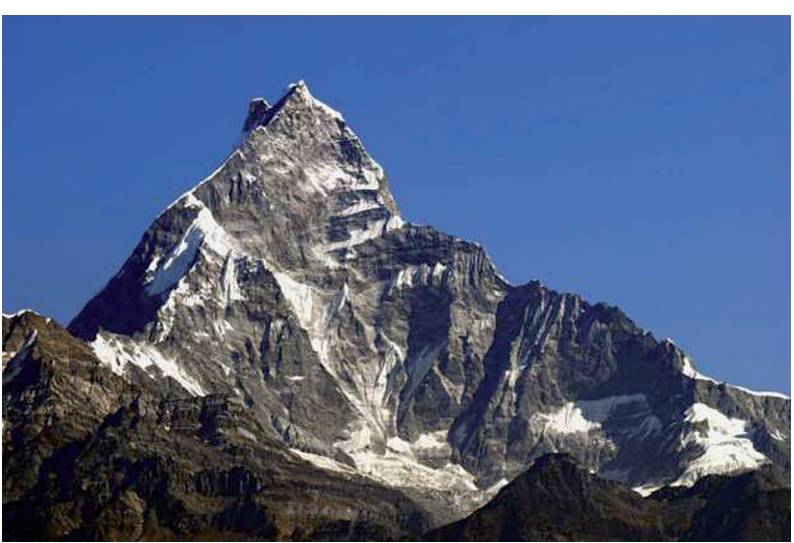

Fig. 7. The sacred "Fishtail" mountain Machhapuchhare, Nepal Himalaya

Source: Wikimedia Commons.

between these peaks and peaks in the contiguous US (Mount Saint Elias does, however, come close to the top five, and actually beats Everest, despite being over 3000 meters lower.)

\section{Reduced ORS (RORS)}

It is common to see a list of the highest $N$ peaks in a region, for example, the top $53^{13}$ peaks in Colorado, known as the "fourteeners" - the peaks exceeding 14,000 feet. Such a list is often interpreted as comprising the "best", "most impressive", or most desirable (for a climber) peaks in the region. We would like to make such

13 The exact number depends on exactly what list is used; see [8]. a list using ORS, which would give more credit to locally high and steep peaks, not simply the highest ones above sea level. However, we need something more than ORS to do so. Note that even standard height-based lists must include some sort of cutoff criterion, to avoid listing trivial subpeaks (or, in the logical extreme, an infinity of points surrounding the summit of the highest peak on the list). Some measures, notably topographic prominence [5], need no such cutoff; such a measure automatically factors in the independence of a summit, meaning that no trivial subpeak will get a high value. We created reduced ORS (RORS) to have this feature: it is a measure of a summit' $\mathrm{s}^{14}$ independent impressiveness. In particular, its most important property is that it is automatically discrete: informally, if a point $P$ gets a high value for RORS, then all nearby points will get a low value. However, our particular definition of RORS involves more choices than we made for ORS, some of which are justified more on aesthetic than mathematical grounds.

To fix ideas and motivate the definition of RORS, consider the Teton Range in Wyoming. It is dominated by the Cathedral Group, with highest point the summit of the Grand Teton (4197 m), shown in Fig. 8 and mapped in Fig. 9. There are other massifs in this small range, most notably that of Mount Moran, a few miles north of the Grand Teton; see Fig. 10.

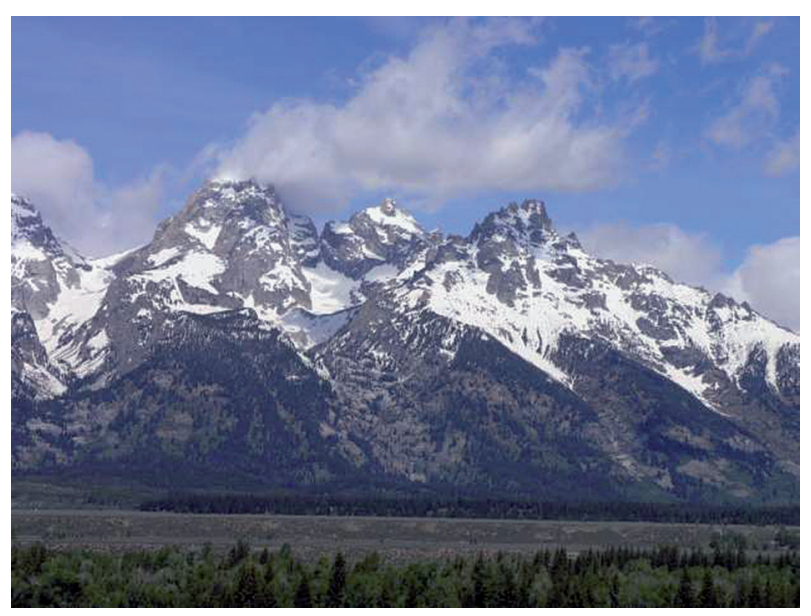

Fig. 8. The Cathedral Group, Grand Teton on the left, Mount Owen center, Teewinot Mountain right Source: Wikimedia Commons.

14 Actually, RORS, like ORS, can be applied to any point on a landscape. However, the points with large RORS values tend to be (but are not always) summits. 


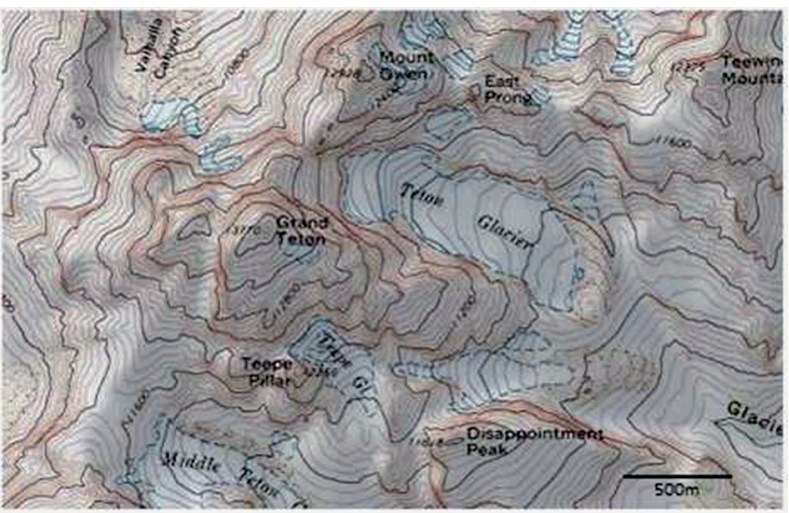

Fig. 9. Topographic map of the Cathedral Group, including the Grand Teton and Mount Owen Source: USGS.

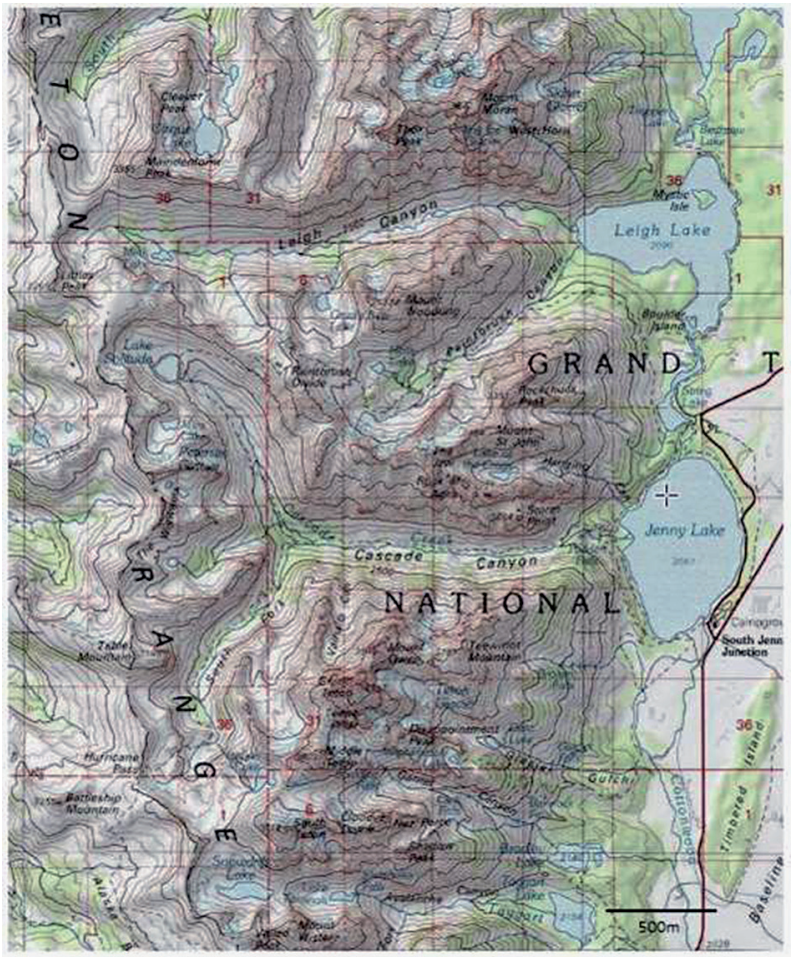

Fig. 10. Zoomed-out map of the Teton range, Mount Moran near the top Source: USGS.

The point with the highest ORS value (ORS $=683 \mathrm{~m})$ is the summit of the Grand Teton. See the topographic map (Fig. 9), or better, view the range in Google Earth. Hence, if we were to make a list of the "best" points in the range as judged by ORS, that summit would clearly top the list. But what should be number two? Certainly not the second-highest boulder on the same summit, and perhaps not even nearby peaks such as Mount Owen (3940 m, just north of the Grand Teton, in the center of Fig. 8), or Teewinot Mountain (3758 $\mathrm{m}$, on the right in the same picture), which are overshadowed significantly by their neigh- bor, and which could reasonably be considered subsidiary points on the same massif. We would like the RORS value of Mount Owen, for example, to be substantially reduced, compared to its ORS value of $581 \mathrm{~m}$, by the presence of the Grand Teton nearby. One way to say this is that, given that the Grand Teton has a high ORS value, the fact that Mount Owen also has a high ORS value does not convey that much new information, since Mount Owen is part of the same massif. The RORS value of Mount Owen is supposed to reflect, roughly, the relief and steepness that it has apart from its being a part of the Grand Teton massif. The same is true of Teewinot Mountain (ORS $=552 \mathrm{~m})$, although since it is substantially more separate from the Grand Teton than Mount Owen is, it should be less reduced by the presence of the Grand Teton. In fact, the number two on the RORS-ranked list (explained in detail below) for the Teton Range (and for all of Wyoming) is Mount Moran, which is significantly more independent than Mount Owen or Teewinot Mountain (see Fig. 10).

To explain RORS in more detail, we continue with the example of the Teton Range. There are a few steps to get to our goal of a measure that will produce the kind of ranked list of independent peaks that we want. First we define relative RORS as follows (details in the appendix). Suppose we want to consider a subpeak of Mount Moran, say Thor Peak (see Fig. 10), and calculate its RORS value considering its proximity only to Mount Moran, which we will call the "reducing" peak in this situation. We denote that value by RORS(Thor; Moran). To calculate it, roughly speaking, for every point in the surrounding terrain, we calculate how impressive the summit of Thor looks from that point, then subtract the impressiveness of Moran from that number (flooring at zero), and then sum to get RORS(Thor; Moran). We could also reduce Thor by both the Grand Teton and Moran to get a smaller value, RORS(Thor; Grand, Moran). In fact, given any chosen collection of reducers, say $\mathbf{p}_{1^{\prime}}, \ldots, \mathbf{p}_{\mathrm{n}^{\prime}}$ we can define the relative RORS of a peak $\mathbf{p}_{0^{\prime}}$, reduced by $\mathbf{p}_{1^{\prime}}, \ldots, \mathbf{p}_{\mathrm{n}^{\prime}}$ and denote it by RORS $\left(\mathbf{p}_{0^{\prime}} ; \mathbf{p}_{1^{\prime}}, \ldots, \mathbf{p}_{\mathrm{n}}\right)$. This number measures the impressiveness that $\mathbf{p}_{0}$ has independent of its association with $\mathbf{p}_{1}, \ldots, \mathbf{p}_{\mathrm{n}}$. Similarly, we could calculate RORS(Owen; Grand), RORS(Teewinot; Grand), RORS(Owen; Teewinot, Grand) or RORS (Teewinot; Owen, Grand). 
Table 3. Top 10 peaks in Wyoming by RORS

\begin{tabular}{|l|c|c|c|c|c|}
\hline \multicolumn{1}{|c|}{ Peak } & ORS & RORS & Elev & Lat & Long \\
\hline Grand Teton & 683 & 683 & 4197 & 43.7413 & -110.8024 \\
\hline Mount Moran & 570 & 531 & 3842 & 43.8379 & -110.7729 \\
\hline Squaretop Mountain & 445 & 444 & 3565 & 43.2246 & -109.7899 \\
\hline P11445 & 353 & 347 & 3489 & 43.2032 & -109.7301 \\
\hline Mount Woodring & 389 & 326 & 3533 & 43.8038 & -110.7935 \\
\hline Temple Peak & 315 & 314 & 3954 & 42.6985 & -109.1710 \\
\hline Fremont Peak & 313 & 308 & 4190 & 43.1263 & -109.6213 \\
\hline Forlorn Pinnacle & 311 & 308 & 3548 & 43.3201 & -109.7735 \\
\hline Lizard Head Peak & 316 & 307 & 3914 & 42.7901 & -109.1976 \\
\hline
\end{tabular}

Relative RORS leads to a wealth of numbers measuring relative impressiveness. However, the most common use we have for RORS is to create a ranked list of peaks ${ }^{15}$ in a region, which we do as follows. We first find the peak with the highest ORS value; call it $\mathbf{p}_{1}$. That will be number one on the list. Then we re-scan the region, evaluating $\operatorname{RORS}\left(\mathbf{p}_{0} ; \mathbf{p}_{1}\right)$ for all points $\mathbf{p}_{0}$. The peak with the highest value becomes the second peak on the list; call it $\mathbf{p}_{2}$. We again scan the region, calculating RORS $\left(\mathbf{p}_{0} ; \mathbf{p}_{1}, \mathbf{p}_{2}\right)$ for all $\mathbf{p}_{0} ;$ the best peak found becomes $\mathbf{p}_{3^{\prime}}$ and so on. This yields a list with the property that the $n$th entry is the best among all points in the region when considered relative to the points above it on the list. We denote the resulting RORS values simply by $\operatorname{RORS}\left(\mathbf{p}_{1}\right)$, $\operatorname{RORS}\left(\mathbf{p}_{2}\right)$, etc., suppressing the dependence on the reducing peaks, since they are implied by the ordering of the list. This "absolute" version of RORS is what we have calculated numerous examples of on our website [7].

As an example of a RORS-ranked list, Table 3 shows the top 10 peaks in Wyoming, headed by the Grand Teton. Here ORS, RORS, and elevation are in meters. Note that Teewinot Mountain appears at the bottom of the list, drastically reduced by the Grand Teton, but not so drastically as the higher (but much closer to the Grand) Mount Owen. The RORS value for Mount Owen is in fact under $150 \mathrm{~m}$, well off the list. It is so low partly because Mount Owen is reduced not only by the Grand Teton but also by Teewinot Mountain; together they reduce the relative impressiveness of Mount Owen in almost all directions. A KML file for this list is available at www.peaklist.org.

15 Or features; sometimes (such as with El Capitan) the best points are not summits. However, for simplicity we will speak of "peaks".
If done by the letter, this procedure is obviously cumbersome, especially if we want a long list of best peaks. However, it is easy to make approximations and simplifications that reduce the time required to compute RORS significantly.

First, since RORS $\leq$ ORS, one need not consider points that do not have a relatively high ORS value. Second, since the effect of reduction falls off relatively quickly with distance, one need not include far-away peaks as potential reducers. Third, reducing by many points almost never produces much more reduction than reducing by the most "powerful" (usually the closest) two or three reducing points.

Nonetheless, calculating the top 50 points by RORS in a US state, for example, is a compute-intensive process. It is also somewhat sensitive to small errors in the data, but that is unavoidable for a measure of this type - RORS is a "winnertakes-all" measure, where two peaks that are close to being tied (and close physically to each other) can get forcibly separated on the list, with one being declared the winner, and the other getting drastically reduced by the winner (as in the case with the Grand Teton and Mount Owen). In the appendix we discuss how this type of sensitivity is unavoidable.

Table 4 shows the top 10 peaks in the Contiguous US, ranked by RORS. Note the mixture of Cascade stratovolcanoes (large relief, not very steep) with rock peaks (smaller but steeper) in the Rockies (mostly Glacier National Park), North Cascades (e.g. Hozomeen Mountain, Fig. 11), and Sierra Nevada (just Half Dome, a classic example of a feature rewarded by ORS/RORS). Mount Index (Fig. 12), also in the North Cascades, is particular striking in terms of its ratio of RORS to elevation; it rises extremely steeply from close to the flatland around Puget Sound. It is a famous 
Table 4. Top 10 peaks in Contiguous US by RORS

\begin{tabular}{|r|l|l|c|c|c|}
\hline Rank & \multicolumn{1}{|c|}{ Peak } & \multicolumn{1}{c|}{ Region } & ORS & RORS & Elev \\
\hline 1 & Mount Rainier & Cascade & 827 & 827 & 4301 \\
\hline 2 & Grand Teton & Teton & 683 & 683 & 4197 \\
\hline 3 & Mount Shasta & Cascade & 675 & 675 & 4317 \\
\hline 4 & Mount Cleveland & Glacier NP & 672 & 672 & 3190 \\
\hline 5 & Mount Hood & Cascade & 649 & 649 & 3452 \\
\hline 6 & Hozomeen Mountain & N Cascade & 643 & 642 & 2440 \\
\hline 7 & Kinnerly Peak & Glacier NP & 612 & 605 & 3035 \\
\hline 8 & Mount Stimson & Glacier NP & 590 & 588 & 3069 \\
\hline 9 & Mount Index & N Cascade & 588 & 583 & 1598 \\
\hline 10 & Half Dome & Sierra Nevada & 580 & 580 & 2692 \\
\hline
\end{tabular}

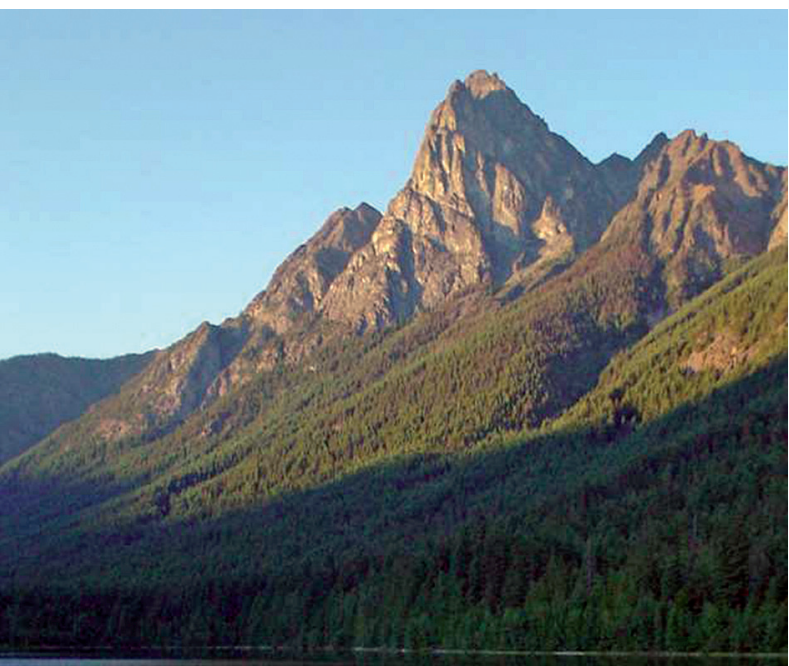

Fig. 11. Hozomeen Mountain, North Cascades, Washington Source: Wikipedia.

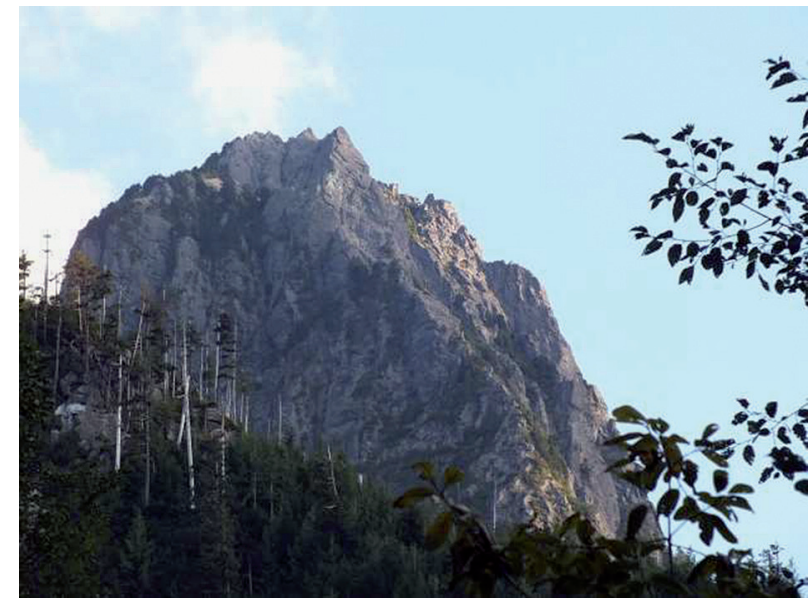

Fig. 12. Mount Index, North Cascades, Washington Source: Wikipedia.

technical climb, but no listing of peaks by elevation (even just in Washington State) would ever include it.

Note that for most of these peaks RORS is very close to ORS, since they are the best peak in their neighborhood. This may seem to make RORS pointless in this situation, but remember that a list purporting to show the "top 10 by ORS" in the Contiguous US would be nonsense; it would be 10 points on Mount Rainier. Also note that by ORS, Mount Owen would (just barely) make this list, while by RORS it is not even close.

\section{Domain relief and steepness (DRS)}

Since ORS is based on a combination of relief and steepness, it is very well adapted to be the basis of a measure of ruggedness. We call this measure domain relief and steepness (DRS). The DRS of a region, let's say a bounded domain $K$, is actually quite a bit simpler to define precisely than RORS. Essentially, it is the RMS average of the ORS value for every point in $K$. There are two modifications to that statement: first, we redefine ORS to integrate over only $K$, since the ruggedness of a given region should not depend on how it sits relative to its surroundings; it is a purely internal measurement. Second, instead of declaring our modified slope integrand $f$ from equation (1) to have $f(u)=0$ for $u<0$, we extend it as an even function. ${ }^{16}$

Denoting this modification of ORS by ORS(p; $h, K)$ we define

$$
\operatorname{DRS}(h, K)=\left[\frac{1}{A(K)} \int_{K}(\mathrm{ORS}(\mathbf{p} ; h, K))^{2} d A(\mathbf{p})\right]^{1 / 2}
$$

where $A(K)$ is the area of $K$. (If $A(K)=0$, we define $\operatorname{DRS}(h, K)=0$; we will justify this in the appendix.) This can be expressed directly in terms of

16 This second change is not essential, but it does make the resulting formula more symmetric. It is easy to verify that using the original convention for $f$ instead results in a definition of DRS that is $1 / \sqrt{ } 2$ times that given here. 
the (new) modified slope integrand $f$ as follows. Abusing notation slightly, let $u(\mathbf{p}, \mathbf{x})=(h(\mathrm{p})-h$ (x)) / $\|\mathbf{p}-\mathbf{x}\|$ be the slope between the points $\mathbf{p}$ and $\mathbf{x}$. Then

$$
\operatorname{DRS}(h, K)=\left[\frac{1}{A(K)} \int_{K \times K}(f(u(\mathbf{p}, \mathbf{x})))^{2} d A(\mathbf{p}) d A(\mathbf{x})\right]^{1 / 2}
$$

Note that this (quadruple) integral is symmetric in the variables $\mathbf{p}$ and $\mathbf{x}$, and that it has units of length, just as ORS does (recall that $f$ is dimensionless).

As with RORS, this version of DRS is not exactly what we are most interested in. Given a mountain range, it is not usually clear what its exact "natural" boundaries are, so the region $K$ is not usually given to us from the start (unless we specify it to be some adjudicated region such as a state or county). What could presumably be more interesting is to let DRS itself define a kind of natural boundary, by starting with a large region $K_{0}$ that clearly includes the whole range and looking for the region $K$ inside $K_{0}$ with the largest DRS value. We have not proved rigorously that such a region exists, although all of our computer calculations, with various grid sizes, suggest that it does. However, the more serious issue is that the resulting region $K$ tends to be quite small, and actually picks out the most rugged single feature or massif, or at most a small range of peaks. The reason is that DRS $(h, K)$ tends to be maximized when the size of $K$ (say, a rough diameter) is not a lot larger than the typical scale of relief of $h$ in $K$ (see the Wasatch Range analysis below for an explicit example of this phenomenon.)

Hence we introduce one more parameter, a fixed area $k$, and find the region $K$ within $K_{0}$ that maximizes DRS $(h, K)$ subject to the constraint $A(K)=k^{17}$. Increasing $k$ yields an expanding sequence of regions that go from focusing on the most rugged massif, to capturing the rugged "heart" of the range, to covering the whole range. At each stage the optimal region gives the most rugged terrain encompassable by a region of the chosen size. If desired, one can then choose a value for $k$ that corresponds to one's notion of where the whole range ends. Making comparisons between ranges requires comparing DRS values

17 Again, using "maximize" in an empirical sense, since we lack a precise, rigorous maximization theorem. calculated for regions of similar size: one range may have a very rugged massif inside it and yet be comparatively tame otherwise (such as, for example, the Washington Cascades around Mount Rainier), while another range may be moderately rugged over a much larger area. Better, a graph of the optimal DRS values for a range as a function of $k$ captures at a glance both the ruggedness and scale of the range as in the following example.

Our main example is the Wasatch Range in Utah, summarized by Table 5 and Fig. 13. The first entry in the table is (a good approximation of) the overall maximizer for DRS, not constrained to have a particular area value. (Hence if we continued the graph in Fig. 13 further to the left, it would decrease again.) Note that it focuses on less than one whole mountain (Fig. 14). The other entries are optimal for their given areas ${ }^{18}$, and they gradually encompass more and more of

Table 5. Optimally rugged regions in the Wasatch Range

\begin{tabular}{|l|r|c|c|}
\hline \multicolumn{1}{|c|}{ Description } & $\begin{array}{c}\text { Size } \\
\left(\mathrm{km}^{2}\right)\end{array}$ & $\begin{array}{c}\text { DRS } \\
(\mathrm{m})\end{array}$ & Figure \\
\hline $\begin{array}{l}\text { North Face Cascade } \\
\text { Mountain }\end{array}$ & 10.9 & 160 & 14 \\
\hline $\begin{array}{l}\text { Timpanogos/ Cascade } \\
\text { Mountain }\end{array}$ & 31.8 & 155 & 15 \\
\hline $\begin{array}{l}\text { Above plus Little } \\
\text { Cottonwood }\end{array}$ & 613.0 & 121 & 16 \\
\hline $\begin{array}{l}\text { Above plus Big } \\
\text { Cottonwood, Mount Nebo }\end{array}$ & 1390.0 & 109 & 18 \\
\hline $\begin{array}{l}\text { Wasatch Front, Mill Creek } \\
\text { to Mount Nebo }\end{array}$ & 3440.0 & 89 & 19 \\
\hline $\begin{array}{l}\text { Wasatch Front, Wellsville } \\
\text { Mountains to Mount Nebo }\end{array}$ & & \\
\hline
\end{tabular}

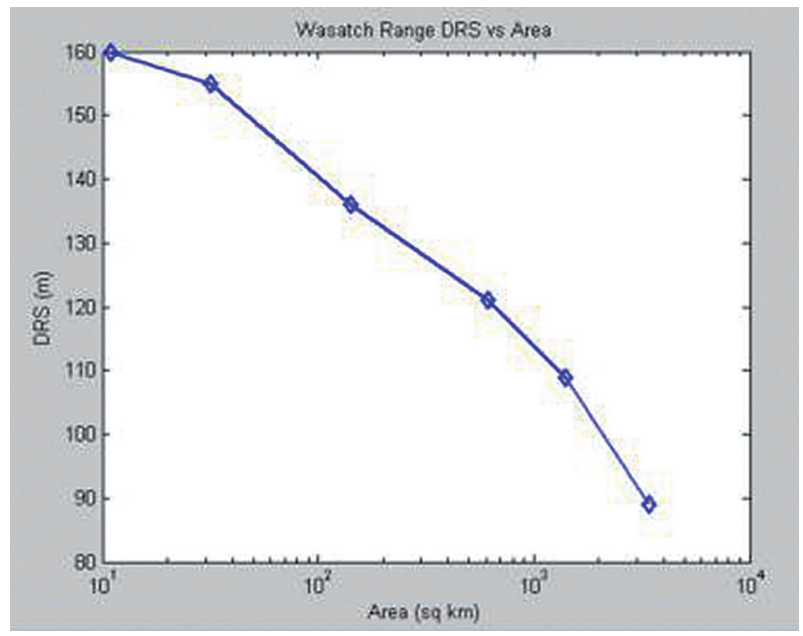

Fig. 13. Graph of optimal DRS values as a function of area, for the Wasatch Range in Utah

18 To see an explanation of the area values chosen, see the Appendix. 


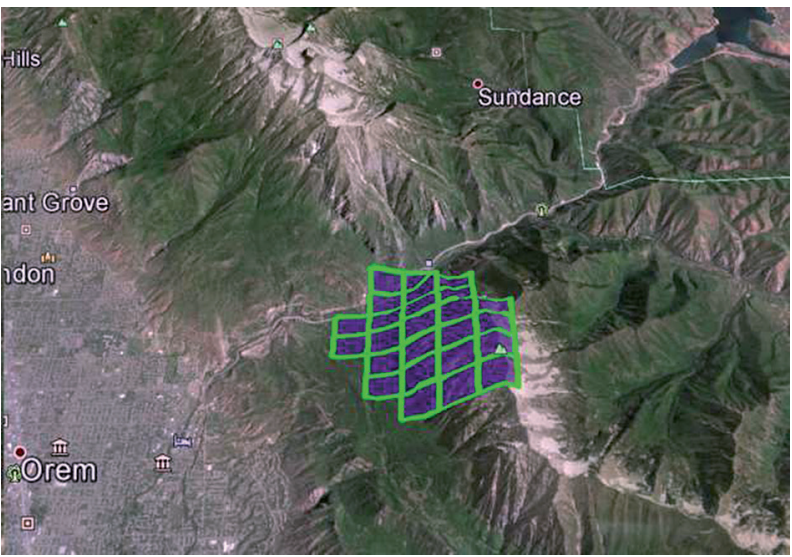

Fig. 14. Wasatch Range, overall maximizer for DRS Basemap: Google Earth.

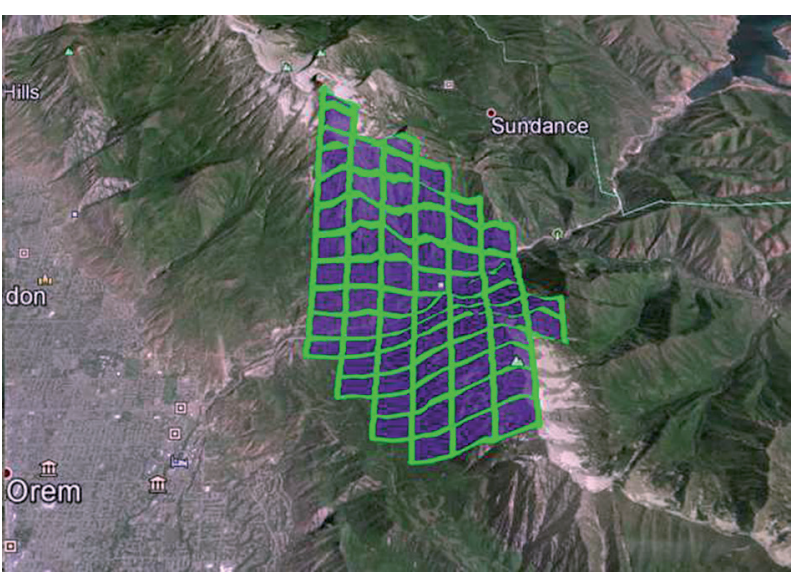

Fig. 15. Wasatch Range, maximizer for $31.8 \mathrm{sq} \mathrm{km}$ Basemap: Google Earth.

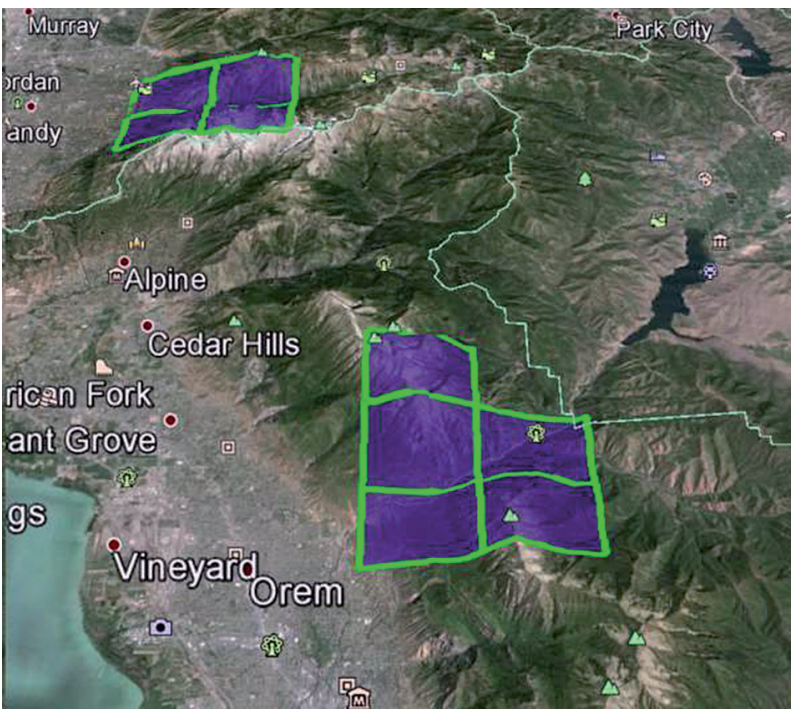

Fig. 16. Wasatch Range, maximizer for $141 \mathrm{sq} \mathrm{km}$ Basemap: Google Earth.

the range, always focusing on the most rugged parts.

In Fig. 20, we show a comparison of graphs of DRS versus area for some of the world's most rugged ranges. Remember that a rough way to

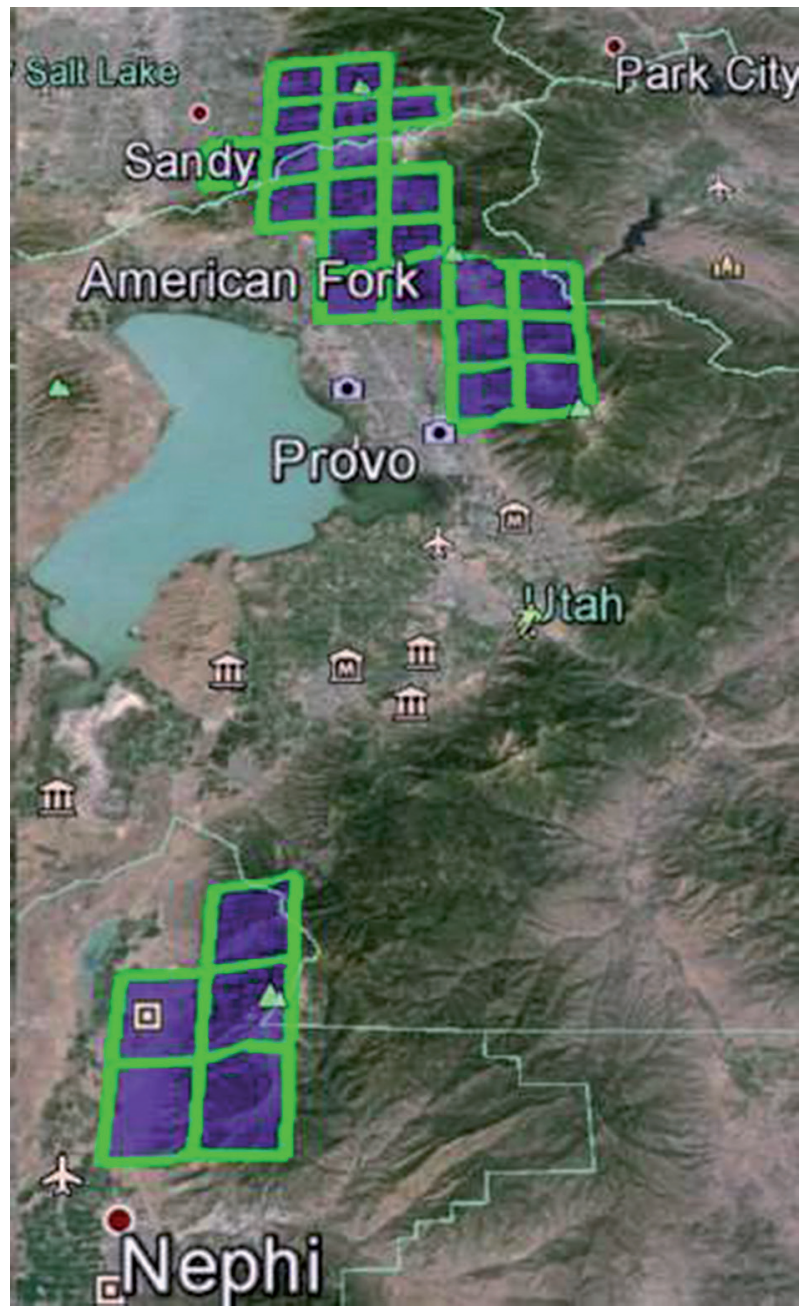

Fig. 17. Wasatch Range, maximizer for $613 \mathrm{sq} \mathrm{km}$ Basemap: Google Earth.

think about a DRS value is as the average ORS value over the given region, and that an ORS of 400 , say, is achieved by a $45^{\circ}$ cone of height 800 meters (or to use a real-world example, Mount Nebo in the Wasatch Mountains). So the Nepal Himalaya and the Karakoram, when restricted to their best 10,000 square kilometers, have an "average impressiveness" equivalent to the summit of such a peak. ${ }^{19}$

Note that the Nepal Himalaya and the Karakoram are very comparable in ruggedness, over the same range of sizes, and are substantially more rugged than the other three ranges shown. The Pamir Range is superior to the Alps and the Canadian Coast Mountains at the smaller scale,

19 Due to using the even extension of the function $f$ in the definition of DRS, we count valley terrain that looks up at nearby steep peaks as impressive, along with peaks that look down at nearby valleys. 


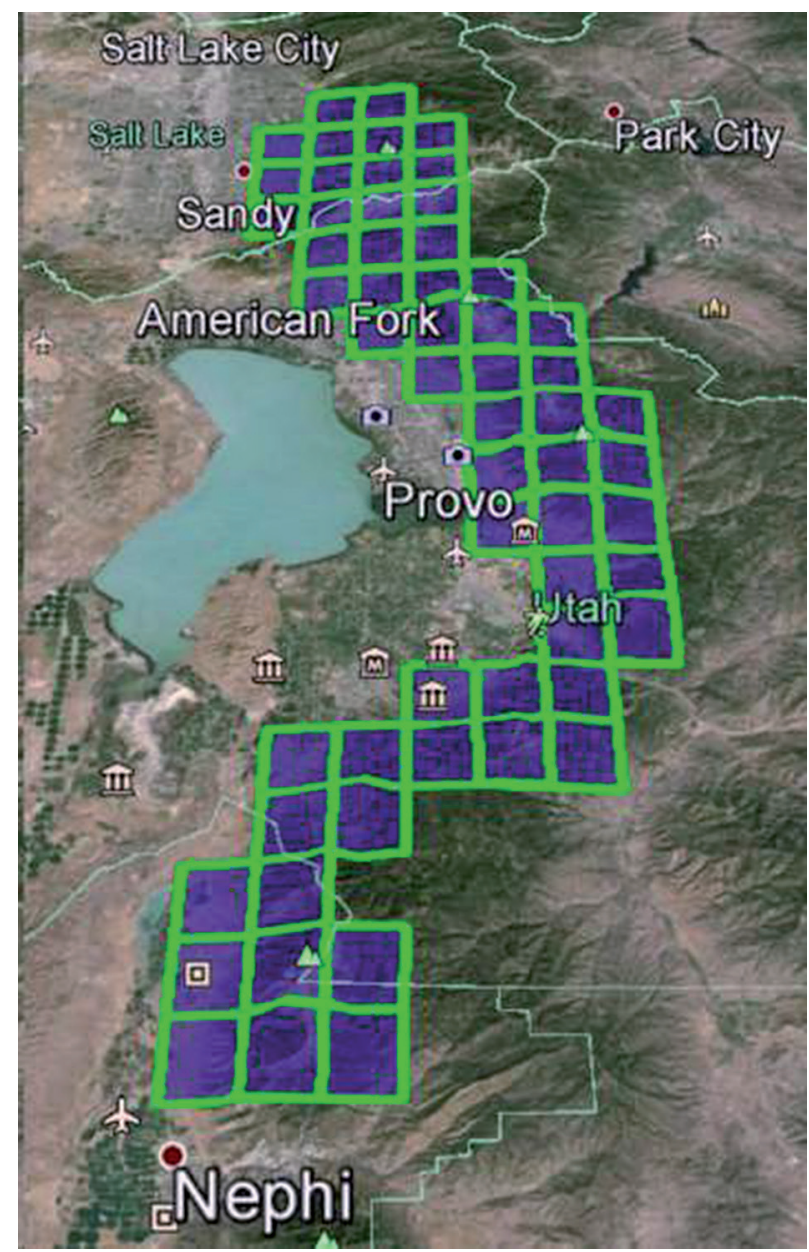

Fig. 18. Wasatch Range, maximizer for $1390 \mathrm{sq} \mathrm{km}$ Basemap: Google Earth.

but is quite comparable to those two when the whole range is taken into account, even though the Pamir Mountains reach over 7000 meters, while the Coast Mountains have very few summits over 3000 meters.

Recent advances in digital mapping will make it possible to eventually calculate reasonably accurate ORS, RORS, and DRS values for any region on the planet (except perhaps the polar regions). We look forward to sharing the resulting comparisons, and use the values to identify interesting mountain terrain.

Appendix: Precise definitions and proofs of properties of RORS and DRS

\section{RORS}

We first define RORS of a reference point $\mathbf{p}_{0}$ relative to a specific set of points $\mathbf{p}_{\mathrm{i}} \ldots, \mathbf{p}_{\mathrm{n}^{\prime}}$ and a landscape function $h$. One should think of $\mathbf{p}_{0}$ as

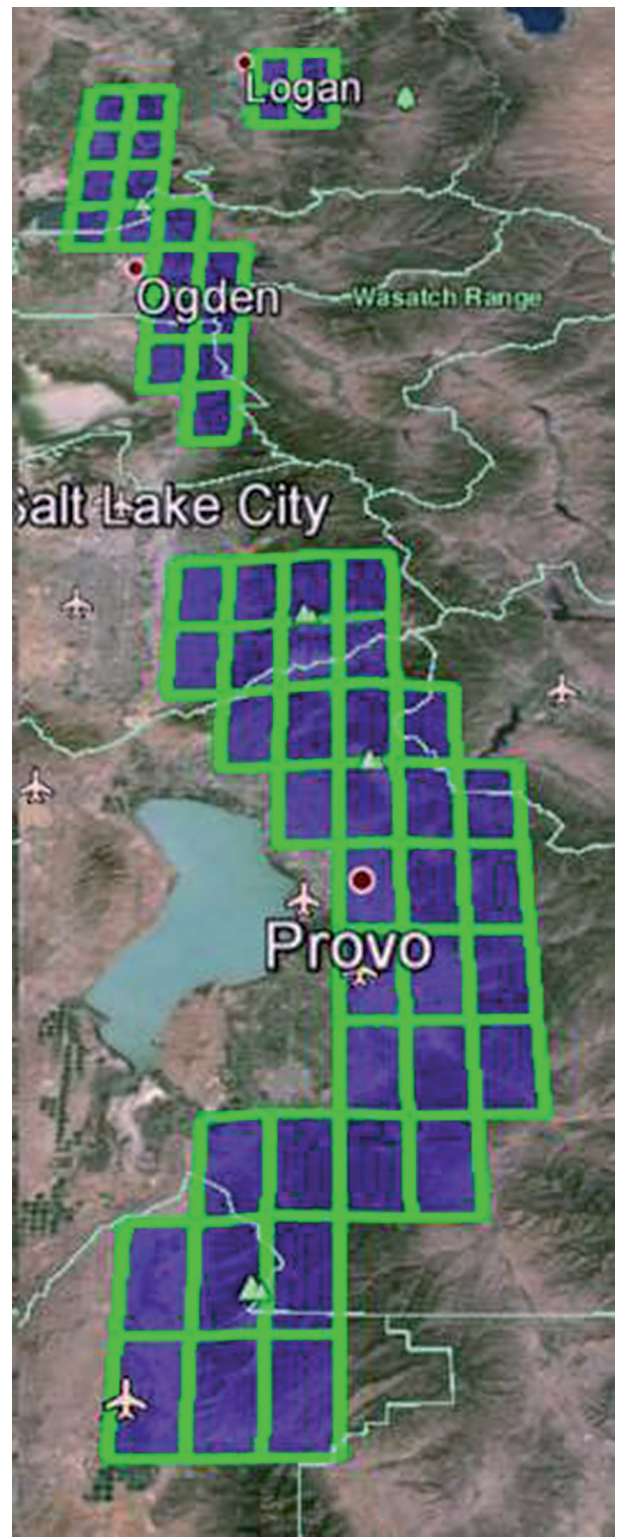

Fig. 19. Wasatch Range, maximizer for $3440 \mathrm{sq}$ km Basemap: Google Earth.

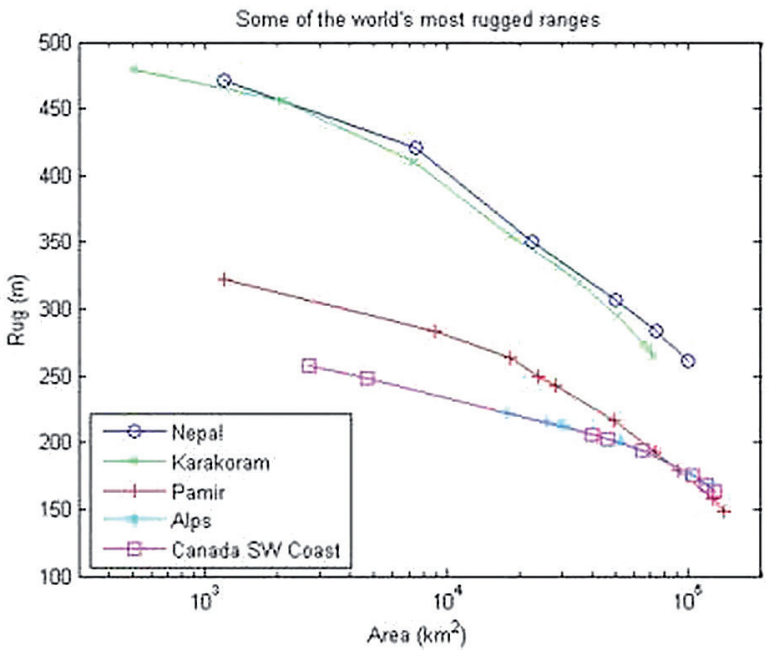

Fig. 20. Ruggedness curves for selected world ranges 
a summit to be evaluated, and $\mathbf{p}_{1}, \ldots, \mathbf{p}_{\mathrm{n}}$ as nearby, more impressive summits. To obtain RORS, we modify the integrand in the definition of ORS so that each sample point contributes only to the extent that "viewing" $\mathbf{p}_{0}$ from $\mathbf{x}$ is "more impressive" than viewing $\mathbf{p}_{1}, \ldots, \mathbf{p}_{\mathrm{n}}$. Precisely, we set

$$
u_{i}(\mathrm{x})=\frac{h\left(\mathbf{p}_{i}\right)-h(\mathrm{x})}{\left\|\mathbf{p}_{i}-\mathbf{x}\right\|} \quad(i=0, \ldots, n)
$$

and

$$
v_{i}(\mathbf{x})=f\left(u_{i}(\mathbf{x})\right)
$$

where

$$
f(u)=\left[\frac{4}{\Pi^{3}}\left(2 u \arctan u-\ln \left(u^{2}+1\right)-\arctan ^{2} u\right)\right]^{1 / 2}
$$

is the modified slope function used in ORS. Then for each $i=1, \ldots, n, v_{0}(\mathbf{x})-v_{i}(\mathbf{x})$ is a measure of the "impressiveness" of the reference point $\mathbf{p}_{0}$ as seen from sample point $\mathbf{x}$, masked, or reduced, by the impressiveness of the point $\mathbf{p}_{1}$. Hence a simple candidate for the new integrand is

$$
\min \left\{\max \left(v_{0}(\mathbf{x})-v_{i}(\mathbf{x}), 0\right)\right\}: i=1, \ldots, n
$$

Note that taking the max with zero prevents negative contributions; once a nearby peak has stolen all of a certain sample point's contribution to $\operatorname{RORS}\left(\mathbf{p}_{0}\right)$, it can't do any more damage. Similarly, using min (instead of, for example, subtracting the sum of the $v_{i}$ ) lets only the most significant detractor act at each sample point. These are choices we make on empirical and practical grounds; one could use other conventions.

We actually perform one more modification on the functions $v_{i}$ before collecting them to build the RORS integrand. To explain this, consider two scenarios. In the first, $\mathbf{p}_{0}$ lies directly between the sample point $\mathbf{x}$ and a better peak $\mathbf{p}_{1}$ (Fig. 21); one can think, for example, of $\mathbf{p}_{0}$ as a subpeak on a ridge of $\mathbf{p}_{1}$, with the sample point at the base of the ridge. In the second scenario, $\mathbf{p}_{0}$ and $\mathbf{p}_{1}$ are diametrically opposed as viewed from $\mathbf{x}$ (Fig. $22)$; for example, they could be on opposite sides of a valley, with the sample point on the valley floor. In the latter scenario, it is plausible to consider $\mathbf{p}_{0}$ as more independent of $\mathbf{p}_{1}$ than it is in the former, due to the relative position of the two peaks as viewed from the sample point.

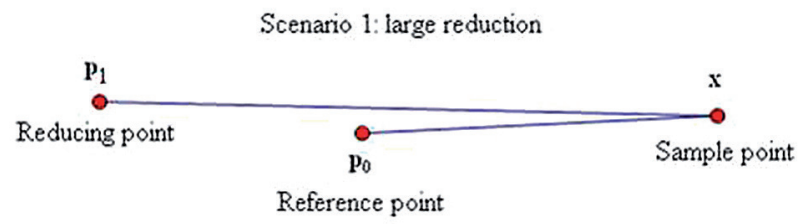

Fig. 21. Scenario 1

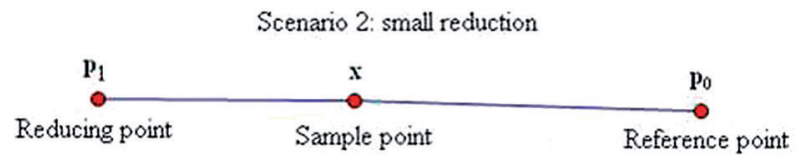

Fig. 22. Scenario 2

You can see these two scenarios in the Swiss Alps in Google Earth: Scenario 1, Scenario 2. To distinguish these situations, we introduce an angle weighting, as follows. For $i=1, \ldots, n$, we let $\alpha_{i}(\mathbf{x})$ be the angle between the rays $\mathbf{x} \mathbf{p}_{0}$ and $\mathbf{x} \mathbf{p}_{\mathbf{i}^{\prime}}$ and we let

$$
w_{i}(\mathbf{x})=\frac{1}{2}\left(1+\cos a_{i}(\mathbf{x})\right)=\cos ^{2}\left(\frac{a_{i}(\mathbf{x})}{2}\right)
$$

Note that $w_{i}$ varies from 1 , in the ridge scenario, down to 0 , in the valley scenario. We then define the RORS integrand $g$ to be

$$
g(\mathbf{x})=\min \left\{\max \left(v_{0}(\mathbf{x})-w_{i}(\mathbf{x}) v_{i}(\mathbf{x}), 0\right): i=1, \ldots, n\right\}
$$

and we define

$$
\operatorname{RORS}\left(\mathbf{p}_{0} ; \mathbf{p}_{1}, \ldots, \mathbf{p}_{n} ; h\right)=\left[\int_{\mathbb{R}^{2}} g^{2}(\mathbf{x}) d A(\mathbf{x})\right]^{1 / 2}
$$

This defines the relative version of RORS; the absolute, list-generating version of RORS is described in the main body of the paper. We denote the absolute RORS of a peak $\mathbf{p}$ simply by $\operatorname{RORS}(\mathbf{p})$.

The one property of RORS that we want to emphasize is that it is automatically discrete: given any cutoff value $C$, the set of points $\mathbf{p}$ in a region with $\operatorname{RORS}(\mathbf{p})>C$ is finite, and the higher $C$ is, the farther these points tend to be from each other. This is in sharp contrast to ORS, which assigns a number to every point of the terrain in a continuous fashion. So, for example, all points near the summit of a large peak will get similarly high 
values of ORS. The drawback of the automatic discreteness of RORS is that it makes it highly sensitive to ties: if two nearby peaks have very similar ORS values (perhaps too close to tell, with the available accuracy, which one is truly better), the RORS ranking procedure will pick one to be the winner, and the other will be drastically reduced. Since this can easily be shown to be the case for any automatically discrete measure, it is a price one has to pay; however it is an impetus to make the RORS calculations as accurate as possible.

\section{DRS}

We first look at some optimization problems for DRS, which inform the discussion in the main body of the paper, but for which we do not yet have rigorous results.

Problem 1: Given a fixed landscape $h$ and a set $K_{0}$ find a subset $K \subset K_{0}$ which maximizes DRS $(h, K)$.

Problem 2: Given a fixed landscape $h$, a set $K_{0^{\prime}}$ and $k>0$, find a subset $K \subset K_{0}$ which maximizes DRS $(h, K)$ subject to the constraint $A(K)=k$.

Problem 1 is a little more natural than Problem 2 , since it lacks the area parameter. Note that the fact that DRS $(h, K) \rightarrow 0$ as $A(K) \rightarrow 0$ (shown below) means that this problem will avoid a simple pathology that is found in most problems of the form "find the region with the greatest average $X^{\prime \prime}$ - usually, a search for such a region will simply converge on the maximum of $X$ on the region. The superadditivity of DRS (also shown below) avoids such a pathology - a small region will always have a small DRS simply because it includes very few sample points. As we noted above, the optimal region does tend to be fairly small - not surprisingly, the horizontal scale of the optimal region approaches (in order of magnitude, at least) the vertical scale.

However, beyond this simple, avoided pathology, there is a much larger problem of whether a minimizing region exists at all, even in Problem 2 , with a fixed area. It is unclear whether, without further assumptions on $K$ or $h$, we will get a sequence of progressively better regions which has no limit (in an appropriate topology). This is of course a classic situation in the calculus of variations, and we have not yet investigated this problem thoroughly. We can lay out a modified problem, as follows.

First, recall the definitions of the $L^{\mathrm{p}}$ norms and $L^{\mathrm{p}}$ spaces.

Definition 1. Given a (Lebesgue measurable) function $f: \mathrm{R}^{2} \rightarrow \mathrm{R}$ and $\mathrm{p} \geq 1$, we define the $L^{\mathrm{p}}$ norm of $f$ by

$$
\|f\|_{p}=\left(\int_{\mathrm{R}^{2}}|f(x)|^{p} d V\right)^{1 / p}
$$

and we define $L^{\mathrm{p}}\left\{f: \mathrm{R}^{2} \rightarrow \mathrm{R}:\|f\| \infty\right\}$. We also define

$$
\|f\|_{\infty}=\inf \{\mathrm{M}: \mathrm{m}\{|\mathrm{f}(\mathrm{x})|>\mathrm{M}\}=0\}
$$

where $m$ denotes Lebesgue measure, and $L^{\infty}=\left\{f: \mathrm{R}^{2} \rightarrow \mathrm{R}:\|f\|_{\infty}<\infty\right\}$. Note that for a continuous function $f,\|f\|_{\infty}$ is just the maximum value of $f$.

Problem 3: Given a fixed landscape $h$, in some class $C \in L^{\infty}$, a class $K$ of allowed regions, and a set $K_{0} \in K$, find a subset $K \subset K_{0^{\prime}}$ with $K \in K$ which maximizes DRS $(h, K)$.

We hope that the classes $C$ and $K$ need not be too restrictive to guarantee a solution. Two examples of our vague thinking along these lines:

Questions: (1) If $h$ is smooth (say $C^{1}$, i.e. continuously differentiable) then can we guarantee a solution to Problem 3, with no a priori restriction on $K$ ? Will the optimal $K$ have a relatively nice boundary? Must we make explicit assumptions about the niceness of the set of critical points of $h$ ? (2) For an arbitrary $h \in L^{\infty}$, if we require $K$ to be convex, can we guarantee a solution to Problem 3 ?

Note that even if one or both of these questions has a positive answer, neither is particularly satisfactory, since both restrictions are rather severe for our setting. Mountain ranges have vertical cliffs, so $h$ is typically not even continuous (although it usually isn't a horribly discontinuous function, so perhaps some sort of piecewise smoothness is an appropriate assumption). And the shape one would expect to get "naturally" (without a priori restriction on $K$ ) for a maximizer would not usually be convex (picture the contours of a mountain range). Our numerical results indicate that without a constraint on the shape of $K$, the optimal region will not necessarily even be connected, much less convex. But both 
questions are reasonable starting points for further investigation.

However, turning from the pure approach to a more applied, numerical approach, we see no signs of any major practical obstacle to solving (approximately) Problems 1 and 2. Coarse-gridded numerical approximations to this problem yield stable results. For example, our calculations indicate that the most rugged region in the contiguous 48 states is the Picket Range of the North Cascades, in Washington State [7].

We will mention one practical note about how we actually proceed with Problem 2 to produce results such as the ones in the main body of this paper. We actually consider a slightly more general form of DRS, namely

$\operatorname{DRS}_{q}(h, K)=\left[\frac{1}{A(K)^{q}} \int_{K} \operatorname{ORS} 2(\mathbf{p}, h(\mathbf{p}) ; h, K) d A(\mathbf{p})\right]^{1 / 2}$

Note that the ordinary case is when $q=1$, and if $q=0$ then we get the "total" $L^{2}$ norm, instead of the "average". So clearly the analog of Problem 1 is silly for the case $q=0$, as the optimal region will always be all of $K_{0}$. But for $0<q<1$, the analog of Problem 1 is just as well-defined as it is for $q=1$, and it will tend to give larger and larger optimal regions as $q$ decreases. It is not much harder to see that adjusting $q$ gives an alternate parametrization to using $\mathrm{A}(K)=k$ for Problem 2. This has proved convenient, as it avoids having to deal with the fixed-area constraint in that problem. So in our calculations presented on the peaklist.org website, we have actually looked for overall max-

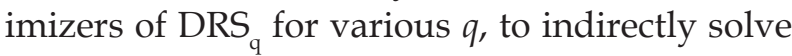
Problem 2.

It may also very well be the case that some DRS $_{q}$ with $q \neq 1$ is of as much or more interest in its own right than DRS $=\mathrm{DRS}_{1}$. It has an extra arbitrary parameter, and we see no clear reason to pick some particular $q \neq 1$, which is why we prefer DRS ${ }_{1}$. But further investigation may make us prefer some other choice of $q$.

Now we turn to careful proofs of some desirable properties of DRS. First, we note that DRS satisfies obvious scaling and invariance properties akin to those satisfied by ORS (see Earl and Metzler 2013). We won't write them down explicitly. Next, we want to give a simple property of DRS which clarifies exactly how much it is like, and how much it is unlike, an ordinary RMS average.
The difference comes from taking sample points only from the region $K$. For an ordinary average, the following inequality would be an equality.

Lemma 1. Let the landscape function $h$ be fixed and suppose $K=K_{1} \cup K_{2}$ with $K_{1}, K_{2}$ disjoint. Then

$\operatorname{DRS}^{2}(h, K) \geq \frac{A\left(K_{1}\right) D R S^{2}\left(h, K_{1}\right)+A\left(K_{2}\right) D R S^{2}\left(h, K_{2}\right)}{A(K)}$

Proof. Let $g(K)=A(K) D R S^{2}(h, K)$. Then

$$
\begin{aligned}
g(K) & =\int_{K^{\prime} \times K} f^{2}(u(\mathbf{p}, \mathbf{x})) d A(\mathbf{p}) d A(\mathbf{x}) \\
& =\int_{K_{1} \times K_{1}} f^{2}(u(\mathbf{p}, \mathbf{x})) d A(\mathbf{p}) d A(\mathbf{x}) \\
& =\int_{K_{2} \times K_{2}} f^{2}(u(\mathbf{p}, \mathbf{x})) d A(\mathbf{p}) d A(\mathbf{x}) \\
& =\int_{K_{1} \times K_{2}} f^{2}(u(\mathbf{p}, \mathbf{x})) d A(\mathbf{p}) d A(\mathbf{x}) \\
& \geq g\left(K_{1}\right) \mid g\left(K_{2}\right)
\end{aligned}
$$

which is what we wanted to show.

We refer to this property as the "superadditivity" of DRS (although more properly it is $g$ which is superadditive).

Proposition 1. DRS is continuous as a function of $h$ in the $L^{\infty}$ norm.

Proof. This is clear since DRS is (the square root of) an integral of $\mathrm{ORS}^{2}$, which is continuous in $L^{\infty}$ (Earl, Metzler 2013)

In fact, DRS is substantially better than this simple proposition indicates, since it averages out the variation in ORS. Hence a tall but skinny feature will contribute only a small amount to DRS. First we prove a useful lemma about the behavior of ORS for a tall feature (in the extreme case, a flagpole) on a region of finite area.

Lemma 2. Fix a region $K$ with A $(K)$ finite, a landscape $h \in L^{\infty}(K)$, and a point $\mathbf{p} \in K$. Then ORS $\left(\mathbf{p}, h_{0} ; h, K\right)$ is $0\left(\sqrt{h_{0}}\right)$ as $h_{0} \rightarrow 1$.

Proof. Without loss of generality we can assume that $\mathbf{p}$ is the origin. By adding a constant to both $h_{0}$ and $h$ we can assume that $h(\mathbf{x}) \geq 0$ for all $\mathbf{x} \in K$. Then 


$$
\begin{aligned}
& \operatorname{ORS}^{2}\left(0, h_{0} ; h, K\right)=\int_{K} f^{2}\left(\frac{h_{0}-h(\mathbf{x})}{r}\right) d A(\mathbf{x}) \\
\leq & \frac{4}{\Pi^{2}} \int_{K}\left|\frac{h_{0}-h(\mathbf{x})}{r}\right| d A(\mathbf{x}) \\
\leq & \frac{4}{\Pi^{2}} \int_{K} \frac{h_{0}}{r} d A(\mathbf{x}) \\
\leq & \frac{4 h_{0}}{\Pi^{2}}\left(\int_{0}^{2 \pi} \int_{0}^{1} \frac{1}{r} \quad \mathrm{rdrd} \theta+\int_{K-B_{1}(0)} \frac{1}{r} d A(\mathbf{x})\right) \\
\leq & \frac{4 h_{0}}{\Pi^{2}}\left(2 \Pi+\int_{K} d A(\mathbf{x})\right) \\
\leq & \frac{4 h_{0}}{\Pi^{2}}(2 \Pi+A(K)) \\
\leq & C h_{0}
\end{aligned}
$$

So

$$
\operatorname{ORS}\left(0, h_{0} ; h, K\right) \leq \sqrt{C} \sqrt{h}_{0}
$$

Proposition 2. DRS is continuous as a function of $h$ in the $L^{1}$ norm, for a fixed region $K$.

Proof. Let $h_{1} \in L^{\infty}(K)$ be a fixed landscape and let $h_{2} \in L^{\infty}(K)$ with $\left\|h_{2}\right\|_{K, 1}<\delta$ for a small $\delta$ with $0<\delta<1$. Let $h=h_{1}+h_{2}$. Let $K_{0}=\left\{\mathrm{p} \in \mathrm{K}: \| h_{2}(\mathbf{p})\right.$ $\|<\sqrt{\delta}\}, K_{1}=K-K_{0}$. Note that $\mathrm{A}\left(\mathrm{K}_{1}\right)<\sqrt{\delta}$ by the condition on the 1-norm of $h_{2}$. So $K_{0}$ is the bulk of $K$, on which $h_{2}$ is actually $L^{\infty}$-small, and $K_{1}$ is a small region on which $h_{2}$ can have large values. We have, once again using the notation $u(\mathbf{p}, \mathbf{x})=$ $|h(\mathbf{p})-h(\mathbf{x})| /\|\mathbf{p}-\mathbf{x}\|$,

$$
\begin{aligned}
D R S^{2}(h, K) & =\frac{1}{A(K)} \int_{K_{1} \times K_{1}} f^{2}(u(\mathbf{p}, \mathbf{x})) d A(\mathbf{x}) d A(\mathbf{p}) \\
& =\frac{1}{A(K)}\left[\int_{K_{0} \times K_{0}} f^{2}(u(\mathbf{p}, \mathbf{x})) d A(\mathbf{x}) d A(\mathbf{p})\right. \\
& +2 \int_{K_{0} \times K_{1}} f^{2}(u(\mathbf{p}, \mathbf{x})) d A(\mathbf{x}) d A(\mathbf{p}) \\
& \left.+\int_{K_{1} \times K_{1}} f^{2}(u(\mathbf{p}, \mathbf{x})) d A(\mathbf{x}) d A(\mathbf{p})\right]
\end{aligned}
$$

Let the three integrals in this expression be denoted $I_{k}\left(h_{2}\right), k=1,2,3$. The bulk of the contribution will be from $I_{1}$. We will show that $I_{1}\left(h_{2}\right)$ is close to $I_{1}(0)$, and that $I_{2}\left(h_{2}\right)$ and $I_{3}\left(h_{3}\right)$ are small, when $\delta$ is small. First, we have

$$
I_{1}\left(h_{2}\right)=\int_{K_{0}} \operatorname{ORS}^{2}\left(\mathbf{p}, h(\mathbf{p}) ; h, K_{0}\right) d A(\mathbf{p})
$$

and, as in the previous proposition, ORS ${ }^{2}$ is $L^{\infty}$-continuous in $h, h$ is $L^{\infty}$-close to $h_{1}$ on $K_{0^{\prime}}$ and $A\left(K_{0}\right)$ is bounded by the fixed finite $A(K)$. Hence $I_{1}\left(h_{2}\right)$ is close to $I_{1}(0)$.

Second, note that on $K_{0^{\prime}}$

$$
\|\mathrm{h}\|_{\mathrm{K}_{0, \infty}} \leq\|\mathrm{h} 1\|_{\mathrm{K}_{0, \infty}} \sqrt{\delta} \leq\|\mathrm{h} 1\|_{\mathrm{K}_{0, \infty}}+1
$$

so

$$
\begin{aligned}
& I_{2}\left(h_{2}\right)=\int_{K_{1}} \operatorname{ORS}^{2}\left(\mathbf{p}, h(\mathbf{p}) ; h, K_{0}\right) d A(\mathbf{p}) \\
& \leq \int_{K_{1}} \operatorname{ORS}^{2}\left(\mathbf{p},|h(\mathbf{p})| ;-\left(\left\|h_{1}\right\|_{K_{0,0}}-1\right), K_{0}\right) d A(\mathbf{p})
\end{aligned}
$$

(by monotonicity)

$$
\leq C_{0} \int_{K_{1}}\left(|h(\mathbf{p})|+\left\|h_{1}\right\|_{K_{0, \infty}}+1\right), d A(\mathbf{p})
$$

(by the $O(\sqrt{h})$ bound from the lemma)

$$
\begin{aligned}
& \leq C_{0} \int_{K_{1}}\left(|h(\mathbf{p})|+2\left\|h_{1}\right\|_{K_{0, \infty}}+1\right), d A(\mathbf{p}) \\
& \leq C_{0}\left\|h_{2}\right\|_{K_{1}, 1}+2 C_{0} A\left(K_{1}\right)\left\|h_{1}\right\|_{K_{K_{\infty}}}+C_{0} A\left(K_{1}\right) \\
& \leq C_{0} \delta+2 C_{0} \sqrt{\delta}\left\|h_{1}\right\|_{K_{1 \infty}}+C_{0} \sqrt{\delta}
\end{aligned}
$$

which is small when $\delta$ is small.

Third, for a small $a>0$ to be determined later, let $S_{1}=\left\{(\mathbf{x}, \mathrm{p}) \in K_{1} \times K_{1}:|\mathbf{x}-\mathrm{p}| \mid>a\right\}$ and let $S_{2}=K_{1} \times K_{1}-S_{1}$. This separates things into the close-interaction regime and the distant regime. 
Then

$$
\begin{aligned}
& I_{3}\left(h_{2}\right)=\int_{K_{1} \times K_{1}} f^{2}(u(\mathbf{p}, \mathbf{x})) d A(\mathbf{x}) d A(\mathbf{p}) \\
& \leq \frac{4}{\pi^{2}} \int_{K_{1} \times K_{1}} \frac{|h(\mathbf{x})-h(\mathbf{p})|}{\|\mathbf{x}-\mathbf{p}\|} d A(\mathbf{x}) d A(\mathbf{p}) \\
& \leq \frac{4}{\pi^{2}} \int_{K_{1} \times K_{1}} \frac{\mid h(\mathbf{x})+h(\mathbf{p})\rfloor}{\|\mathbf{x}-\mathbf{p}\|} d A(\mathbf{x}) d A(\mathbf{p}) \\
& \leq \frac{8}{\pi^{2}} \int_{K_{1} \times K_{1}} \frac{|h(\mathbf{p})|}{\|\mathbf{x}-\mathbf{p}\|} d A(\mathbf{x}) d A(\mathbf{p}) \\
& \leq \frac{8}{\pi^{2}}\left(\int_{S_{1}} \frac{|h(\mathbf{p})|}{a} d A(\mathbf{x}) d A(\mathbf{p})+\right. \\
& \left.+\int \frac{|h(\mathbf{p})|}{\|\mathbf{x}-\mathbf{p}\|} d A(\mathbf{x}) d A(\mathbf{p})\right) \\
& \leq \frac{8}{\pi^{2}}\left(\frac{A\left(K_{1}\right)}{a} \int_{K_{1}}|h(\mathbf{p})| d A(\mathbf{p})+\right. \\
& \left.+\int_{S_{1}}|h(\mathbf{p})| \int_{0}^{2 \pi} \int_{0}^{a} \frac{1}{r} r d r d \theta d A(\mathbf{p})\right) \\
& =\frac{8}{\pi^{2}}\left(\frac{A\left(K_{1}\right)}{a}+2 \pi \mathrm{\alpha}\right) \int_{K_{1}}|h(\mathbf{p})| d A(\mathbf{p}) \\
& =\frac{8}{\pi^{2}}\left(\frac{A\left(K_{1}\right)}{a}+2 \pi \mathrm{a}\right)\left\|h_{1}+h_{2}\right\|_{K_{1}, 1} \\
& =\frac{8}{\pi^{2}}\left(\frac{A\left(K_{1}\right)}{a}+2 \pi \alpha\right)\left(\left\|h_{1}\right\|_{K_{1}, 1}+\left\|h_{2}\right\|_{K_{1}, 1}\right) \\
& =\frac{8}{\pi^{2}}\left(\frac{A\left(K_{1}\right)}{a}+2 \pi \mathrm{\alpha}\right)\left(\left\|h_{1}\right\|_{K_{1}, \infty} A\left(K_{1}\right)+\left\|h_{2}\right\|_{K_{1}, 1}\right) \\
& =\frac{8}{\pi^{2}}\left(\frac{\sqrt{\delta}}{a}+2 \pi \alpha\right)\left(\left\|h_{1}\right\|_{K, \infty} \sqrt{\delta}+\delta\right)
\end{aligned}
$$

Letting $a=\sqrt[4]{\delta}$ yields

$$
I_{3}\left(h_{2}\right) \leq C_{1} \delta^{3 / 4}
$$

which is small when $\delta$ is small. This completes the proof.

Remark. (1) Note that the $L^{1}$-continuity of DRS, which is usually defined only on landscapes in $L^{\infty}$, means that it can be extended to landscapes in $L^{1}$. However we won't make use of that fact. (2) Note that the bounds obtained in the proof are of the form $\sqrt{\delta}$ (or better), which is not enough to show Lipschitz (or similar) behavior. However, we made some fairly drastic approximations in the proof. It may be possible with more finesse to prove that DRS is $L^{1}$-Lipschitz, or at least has good Gateaux derivative behavior with respect to $L^{1}$. (3) Whatever the optimal result may be, even $L^{\infty}$ continuity of DRS is significant, since DRS is a measure of ruggedness, which would ordinarily be calculated with derivatives.

We can also look at continuity in the region $K$. We define a metric on the set of bounded measurable regions $K$ by taking the area (Lebesgue measure) of the symmetric difference:

$$
d\left(K, K^{\prime}\right)=m\left(K \Delta K^{\prime}\right)
$$

There is another way to write this metric. Let $X^{K}$ be the characteristic function of $K$. This is in $L^{1}$ exactly when $K$ has finite area. Then it is easy to see that

$$
d\left(K, K^{\prime}\right)=\left\|\chi^{\kappa}-\chi^{\prime}{ }^{\prime}\right\|_{1}
$$

In other words, taking the characteristic function embeds the set of bounded measurable regions isometrically into $L^{1}$.

Proposition 3. Fix a landscape $h$. Then DRS $(h$, $K)$ is continuous as a function of $K$ with respect to the metric $d$.

Proof. If we do not approach regions with zero area, it is enough to show that the function $g(K)=A(K) \operatorname{DRS}^{2}(h, K)$ is continuous. Note that in general,

$d\left(K, K \cup K^{\prime}\right) \leq d\left(K, K^{\prime}\right) \leq d\left(K, K \cup K^{\prime}\right)+d\left(K^{\prime}, K \cup K^{\prime}\right)$

Hence we can assume without loss of generality that $K \subset K^{\prime}$, and we let $L=K^{\prime}-K$. We have

$$
g(K)=\int_{K \times K} f^{2}(u(\mathbf{p}, \mathbf{x})) d A(\mathbf{p}) d A(\mathbf{x})
$$


so

$$
\begin{aligned}
g\left(K^{\prime}\right)-g(K) & =\int_{K^{\prime} \times K^{\prime}} f^{2}(u(\mathbf{p}, \mathbf{x})) d A(\mathbf{p}) d A(\mathbf{x})- \\
& -\int_{K \times K} f^{2}(u(\mathbf{p}, \mathbf{x})) d A(\mathbf{p}) d A(\mathbf{x}) \\
& =\int_{L \times K} f^{2}(u(\mathbf{p}, \mathbf{x})) d A(\mathbf{p}) d A(\mathbf{x})+ \\
& +\int_{K \times L} f^{2}(u(\mathbf{p}, \mathbf{x})) d A(\mathbf{p}) d A(\mathbf{x}) \\
& +\int_{L \times L} f^{2}(u(\mathbf{p}, \mathbf{x})) d A(\mathbf{p}) d A(\mathbf{x}) \\
& \leq 2 \int_{L} \mathrm{ORS}^{2}(\mathbf{p}, h(\mathbf{p}) ; h, K) d A(\mathbf{p})+ \\
& +\int_{L} \mathrm{ORS}^{2}(\mathbf{p}, h(\mathbf{p}) ; h, L) d A(\mathbf{p}) \\
& \leq 2 \int_{L} \mathrm{ORS}^{2}(\mathbf{p}, h(\mathbf{p}) ; h) d A(\mathbf{p})+ \\
& +\int_{L} \mathrm{ORS}^{2}(\mathbf{p}, h(\mathbf{p}) ; h) d A(\mathbf{p}) \\
& 3 d\left(K, K^{\prime}\right)\|h\|_{\infty}^{2} \\
& \\
& \\
& \\
&
\end{aligned}
$$

using the flagpole bound on ORS. Hence $g$ is actually Lipschitz, and DRS is continuous.

Now we just need to show that as $K$ shrinks to zero area, its DRS value (not just $g(K))$ goes to zero. We have

$$
\begin{aligned}
\operatorname{DRS}^{2}(h, K) & =\frac{1}{A(K)} \int_{K \times K} f^{2}(u(\mathbf{p}, \mathbf{x})) d A(\mathbf{p}) d A(\mathbf{x}) \\
& \leq \frac{4}{A(K)} \int_{K \times K}|u(\mathbf{p}, \mathbf{x})| d A(\mathbf{p}) d A(\mathbf{x}) \\
& \leq \frac{4}{\pi^{2}} \sup _{x \in K} \int_{K}|u(\mathbf{p}, \mathbf{x})| d A(\mathbf{p})
\end{aligned}
$$

Now, in polar coordinates centered at $\mathrm{x}$,

$$
\begin{aligned}
|u(\mathbf{p}, \mathbf{x})| & =\frac{|h(\mathbf{p})-h(\mathbf{x})|}{\|\mathbf{p}-\mathbf{x}\|} \\
& =\frac{|h(\mathbf{p})-h(\mathbf{x})|}{r} \\
& \leq 2 \frac{\|h\|_{\infty}}{r}
\end{aligned}
$$

(a.e.). Hence we need to bound

$$
\int_{K} \frac{1}{r} d A(\mathbf{p})
$$

which, for a fixed area $A(K)=k$, is clearly maximized in the case where $K$ is a disc of radius $a=\sqrt{k / \pi}$ centered at $\mathbf{x}$, in which case

$$
\begin{gathered}
\int_{K} \frac{1}{r} d A(\mathbf{p})=\int_{0}^{2 \pi} \int_{0}^{\sqrt{k / \pi}} d r d \theta \\
=2 \sqrt{\pi k}
\end{gathered}
$$

and this is independent of $\mathbf{x}$, so

$$
\operatorname{DRS}^{2}(h, K) \leq \frac{16}{\pi^{2}}\|h\|_{\infty} \sqrt{\pi A(K)}
$$

which clearly shows continuity as $A(K) \rightarrow 0$.

\section{References}

[1] National Geodetic Survey Datasheets, http://www.ngs. noaa.gov/cgi- bin/datasheet .prl

[2] http:/ / mapper.acme.com/ ?ll=39.11778,-106.44472\&z=14 $\& \mathrm{t}=\mathrm{T}$

[3] British Columbia GNIS Query Page, http://www.ilmb. gov.bc.ca/bcgn- bin/bcg10?name $=25218$

[4] http:/ / mapper.acme.com/?ll=57.09150,-132.37101\&z=13 $\& \mathrm{t}=\mathrm{T}$

[5] Topographic prominence, http://en.wikipedia.org/wiki/ Topographic_prominence

[6] Earl E., Metzler D., 2013. A new topographic functional. Solstice: An Electronic Journal of Geography and Mathematics, vol. XXIV, no. 2. Ann Arbor: Institute of Mathematical Geography. http:// www.imagenet.org/

[7] http://www.peaklist.org/spire/

[8] Fourteener, http://en.wikipedia.org/wiki/Fourteener 